\title{
Body size after-effects are adult-like from 11 years onwards.
}

\author{
Batish, A. ${ }^{1}$, Parchment, A. ${ }^{1}$, Tovée, M.J. ${ }^{2}$, Boothroyd, L.G. ${ }^{1 *}$ \\ 1. Department of Psychology, Durham University, Durham, DH1 3LE, UK \\ 2. School of Psychology, University of Lincoln \\ *Author for correspondence: I.g.boothroyd@dur.ac.uk
}

There is considerable evidence that adults' perception of body weight can be manipulated through adaptation paradigms in which participants are visually exposed to multiple bodies at one weight extreme or the other. No study has yet examined how early such effects can be clearly documented. In the current study we ran an identical experimental adaptation test with adults, 11-12 year olds and 14-15 year olds. Participants viewed bodies ranging from a BMI of 11 to $31 \mathrm{~kg} / \mathrm{m}^{2}$ before and after being adapted to bodies with BMIs below 16 or about 30. Results showed that participants adapted to larger bodies showed a significant change in their weight estimates, such that they were less likely to rate larger bodies as overweight, but showed less change in their ratings of slim bodies. This effect was equally evident in all three age groups, suggesting that this aspect of body perception is functionally mature by 11 years of age.

Keywords: body perception; weight; BMI; adaptation; visual diet

A substantial literature has demonstrated that perception of body size can be manipulated by visual exposure to bodies of very low or very high weight. These effects have been demonstrated with regards to body size attractiveness, normality and perceptions of a healthy weight (Boothroyd et al., 2012; Stephen \& Perera, 2014; Glauert, Rhodes,Byrne,Fink \& Grammer, 2009; Stephens, Bickersteth, Mond, Stevenson \& Brooks, 2016; Stephen, Sturman, Stevenson, Mond and Brooks (2018); Robinson \& Kirkham (2014; Winkler and Rhodes (2005). These effects can be apparent after as little as 15 seconds of repetitive exposure (Jiang \& Vartanian, 2016) and are robust against experimental instructions to participants which direct attention to aspects of the body other than weight (Stephen et al., 2016). Furthermore, groups who spontaneously attend more towards low weight than high weight bodies (i.e. those with pre-existing body weight concerns) may show stronger impacts of visual exposure (Stephen et al., 2018). These effects have been argued to reflect an underlying cognitive representation of bodies which is 'updated' by novel visual information, such that the 'prototype' shifts in the direction of that novel information and all bodies are then judged relative to this adjusted baseline. While the majority of publications have assumed (explicitly or implicitly) that there is a central prototype from which all other stimuli are calibrated, data which assessed perceptions of body weight attractiveness across a BMI range from 12 to $42 \mathrm{~kg} / \mathrm{m}^{2}$ found that both British and Chinese participants had perceptions which were more malleable at the upper end of the BMI range, and did not show clear evidence that perceptions had shifted in a linear manner (Boothroyd et al., 2018). As such these collective results above may represent short term changes in the neural clusters coding for bodies of different sizes, higher level affective changes to some bodies over others, or some combination of the two.

All the above research has focused on adult participants, with a strong bias towards undergraduate samples. Research into facial after effects, however, has demonstrated that these effects can be observed for both identity and attractiveness judgements in children as young as 8 years of age (Anzures et al., 2009; Nishimura et al., 2008). Although the underlying neural responses to facial adaptation paradigms is still maturing until at least 10 years of age (Kadosh, Johnson, Henson, Dick \& Blakemore, 2013), broadly speaking the adaptation effects in these two studies did not differ between children and adults. It was the case however, that Anzures et al (2009) had to use stimuli 
with a stronger distortion in children in order to induce variability in perceptions of attractiveness to begin with, while Nishimura et al. found that the children had weaker overall recognition performance. This is broadly consistent with evidence that holistic elements of facial perception such as identity and emotion are not fully mature until late puberty (Johnson et al 2011; Lawrence et al., 2008).

Research into adaptation to body stimuli is largely lacking in children. There is evidence that viewing pictures of ultra-thin fashion dolls may result in preferences for a smaller own-body size in younger but not older - children (Dittmar, Halliwell and Ives, 2006) but this was not replicated by Rice, Prichard, Tiggemann, and Slater (2016). Similarly while studies involving real dolls show more consistent effects on broader, explicit attitudes to thinness/body esteem, they have shown mixed results on visual body ideals (e.g. Anschultz \& Engels, 2010; Jellinek, Myers, \& Keller, 2016; Rice et al, 2016). To our knowledge no study has assessed body size adaptation/after-effects in children or adolescents using the same paradigms as those used with adults.

Perceptions of body shape across development show that a preference for adult-like waist-hip ratios (i.e. smaller in women than in men) emerges in the peri-pubertal period and is mature by approximately 14 years of age (Conolly et al., 2004). This is broadly concordant with evidence of neurological development where data collectively shows that body-selective regions of the brain may be evident by 7 years of age, but show increasingly adult-like activation and connectivity patterns across childhood and adolescence (Fontan et al., 2017; Ross, de Gelder et al. 2014, submitted' although cf Peelen, Glaser et al. 2009, Pelphrey, Lopez et al. 2009). This being the case, we might expect to see changes in behavioural responses to body adaptation paradigms across the second decade of life.

The current study therefore sought to test the effects of visual exposure to high or low weight bodies on perceptions of weight (from very underweight or very overweight) for peri-pubertal children (11- 12 years), mid-adolescents (14-15 years), and young adults (undergraduate students), in order to establish if the experimental impact of visual exposure differed between these groups.

Stimuli utilised in testing perceptions ranged in weight from 11 to $31 \mathrm{~kg} / \mathrm{m}^{2}$; we predicted a roughly linear association between stimulus BMI and perceived weight and so we examined two elements of that linear association. Firstly, we examined changes in the regression estimate for time $x$ condition in the function for perceived weight regressed onto $\mathrm{BMI}$; if there is a change in the central prototype of a body, we would expect to see a shift such that those viewing thinner bodies should see all bodies as a heavier weight at post-test than pre-test, and vice versa. We also looked at the regression estimate for time $\mathrm{x}$ condition $\mathrm{x} \mathrm{BMI}$; if change in perceptions is predominantly localised to the region of the viewed stimuli then we should see that the time $x$ condition effect becomes more evident at higher stimulus BMI levels for those viewing larger bodies (and vice versa). Finally, by including the interaction between these effects and age group, we will be able to assess whether any of these effects are subject to moderation by participant age (e.g. weaker adaptation in younger groups) or whether they are statistically comparable across groups.

\section{Methods}

Participants. Adolescent participants were recruited through a Catholic high school in an area of moderate economic deprivation in North East England. Parental consent was sought from two classes in each of Year 7 and Year 10. Those for whom consent had been received and who were in school on the day of testing completed the study in class groups. In total $4211-12$ year olds and 61 14-15 year olds were tested. A further $8318-25$ year olds were recruited online through the university's Psychology department participant pool and received partial course credit.

Stimuli. Images were drawn from a set of 50 female bodies of known BMI, photographed in a standardised pose and outfit, with faces obscured (Tovee, Maisey, Emery \& Cornelissen, 1999). The 
set consists of 5 bodies in each of 5 BMI ranges (below 15, 15-19, 20-24, 25-30, and above $30 \mathrm{~kg} / \mathrm{m}^{2}$ ). Each image was given a border (red, purple or plain) and horizontally flipped to create additional stimuli in each weight range.

Procedure. In the pre-adaptation phase, participants viewed 21 images on the range of $12-32 \mathrm{~kg} / \mathrm{m}$. They were then randomised into the two conditions; half were presented with 25 images with a BMI below 16 (thin condition) while half were presented with 25 images with a BMI over 30 (large condition), interspersed with 15 images across the full range to obscure the nature of the experiment. Finally participants viewed the same 21 images as at pre-test, interspersed with 20 topup trials of the adaptation phase stimuli. Wherever the same image was presented more than once, a different border/orientation version was utilised. For all trials (test trials and adaptation/top-up trials) participants rated the body's 'health' on a 7-point Likert scale from 'very underweight' to 'very overweight'.

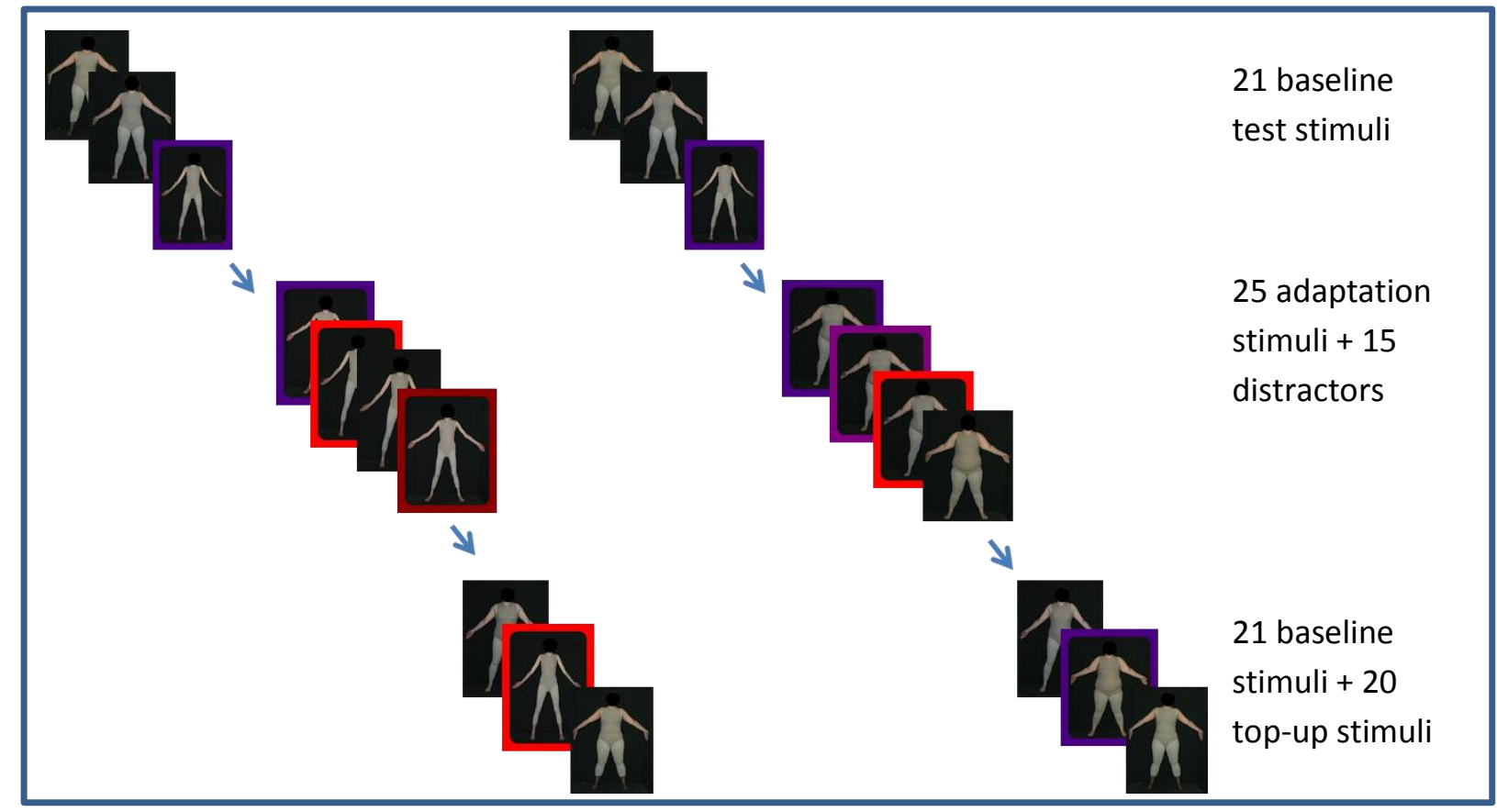

Figure 1. Schematic of experimental design showing thin condition (left) and large condition (right)

\section{Results and Discussion}

Data were analysed in $\mathrm{R}$ ( $\mathrm{R}$ Core Team, 2018) using the Ime4 package (Bates et al, 2015) and stargazer (Hlavac, 2018) in RStudio v1.1.383. Test-trial data were entered into a mixed effects linear model, clustered by participant, with BMI and time as trial-level predictors and sex, age group and condition as participant-level predictors. Results of Models 1-3 are shown in Table 1. Model 1 included gender, age group and image BMI. There was an expected main effect of image BMI such that large images were rated as heavier. There was no effect of gender or age group.

Group, Time and the Group x Time and Group X Time X BMI interactions were added in Model 2 which significantly improved the model. There were main effects we had not predicted of group and time, however, these were likely driven by the interaction effects. There was no group $x$ time interaction effect but crucially there was a significant group $x$ time $x$ BMI effect, such that the higher the BMI of the test stimulus, the lower its weight was rated by participants in the 'large' group at post-test. This is concordant with Boothroyd et al (2018) who found that attractiveness ratings of bodies were likewise more affected by viewing large bodies at the upper end of the BMI range than the lower. As can be seen in Figure 2, the average participant response in the 'thin' condition (in red) produced identical regression functions at pre- and post-test, while the slope is clearly different for the 'large' condition (in blue) with more divergence at the upper weight range. 
Table 1. Regression estimates (with S.E.) for mixed effect linear models testing weight perception of participants for all test images

\begin{tabular}{|c|c|c|c|}
\hline & Model 1 & Model 2 & Model 3 \\
\hline \multicolumn{4}{|l|}{ Gender (female $=$ ref) } \\
\hline Male & $0.001(0.078)$ & $0.018(0.067)$ & $0.020(0.067)$ \\
\hline Other & $-0.179(0.462)$ & $-0.447(0.398)$ & $-0.491(0.397)$ \\
\hline \multicolumn{4}{|l|}{ Age group (11-12yo = ref) } \\
\hline 14-15yo & $0.120(0.092)$ & $0.094(0.079)$ & $0.046(0.081)$ \\
\hline Adult & $0.144(0.090)$ & $-0.004(0.079)$ & $-0.056(0.080)$ \\
\hline Group ('thin' bodies = ref) & & $-0.335^{* * *}(0.061)$ & $-0.348^{* * *}(0.061)$ \\
\hline Time & & $-0.048 * *(0.023)$ & $-0.048 * *(0.023)$ \\
\hline Image BMI & $0.256^{* * *}(0.002)$ & $0.259 * * *(0.002)$ & $0.259 * * *(0.002)$ \\
\hline Group x Time & & $0.115(0.095)$ & $0.127(0.156)$ \\
\hline \multicolumn{4}{|c|}{ Group x Timex Image BMI x Age } \\
\hline $15 y o$ & & & $-0.123(0.205)$ \\
\hline Adult & & & $0.120(0.217)$ \\
\hline Group x Timex Image BMI & & $-0.023 * * *(0.004)$ & $-0.031 * * *(0.007)$ \\
\hline \multicolumn{4}{|c|}{ Group x Timex Image BMI x Age } \\
\hline $15 y o$ & & & $0.015(0.01)$ \\
\hline Adult & & & $0.006(0.01)$ \\
\hline Constant & $\begin{array}{c}-1.350^{* * *} \\
(0.083)\end{array}$ & $\begin{array}{c}-1.126 * * * \\
(0.081)\end{array}$ & $\begin{array}{c}-1.083 * * * \\
(0.082)\end{array}$ \\
\hline Observations & 11,025 & 11,025 & 11,025 \\
\hline Log Likelihood & -14004.06 & -13863.16 & -13855.52 \\
\hline AIC & $28,024.12$ & $27,750.32$ & $27,743.04$ \\
\hline $\mathrm{BIC}$ & $28,082.58$ & $27,838.01$ & $27,859.96$ \\
\hline
\end{tabular}

Adding in the interactions with age group in Model 3 showed no significant interactions and did not meaningfully improve the model. That is to say, the after-effect of viewing large bodies was present in our youngest participants just as much as in our adult participants with no evidence for developmental change between 11 and 25 years. An additional model confirmed that the three-way interaction was present in all three age groups (Supplementary table 1). This strongly suggests that perception of body weight is functionally mature by 11 years of age and aligns with those researchers who argue that other aspects of person perception such as face processing are likewise mature before puberty (e.g. McKone et al., 2009; though cf. Susilo et al., 2013). Our results are also concordant with those of Anzures et al (2009) and Nishimura et al. (2008) who found evidence for facial adaptation in 8 year old children, but unlike these prior studies, we found no overall difference between age groups in terms of their general perceptions of weight.

Our results are somewhat surprising, however, given the evidence that body selective regions of the brain are still developing at 11 years of age. It may be that development in these regions is sufficient by 11 years to show adult-like performance on the current paradigm (see for instance, evidence that some facial perception tasks show adult-like performance at younger ages than others and that adaptation effects may be evident earlier than full facial perception sensitivity; Anzures et al., 2009.) Alternatively, the after-effects observed here may be driven by changes in other regions of the brain, such as those linked to associative and affective associations. As discussed by Boothroyd et al (2018), there is some initial (albeit surprising) evidence that adaptation to body weight and facial attractiveness may both involve left pre-central gyrus (Hummel et al., 2013; Fu et al., 2014), which raises questions about the extent to which adaptation to complex social stimuli like bodies are purely perceptual. 


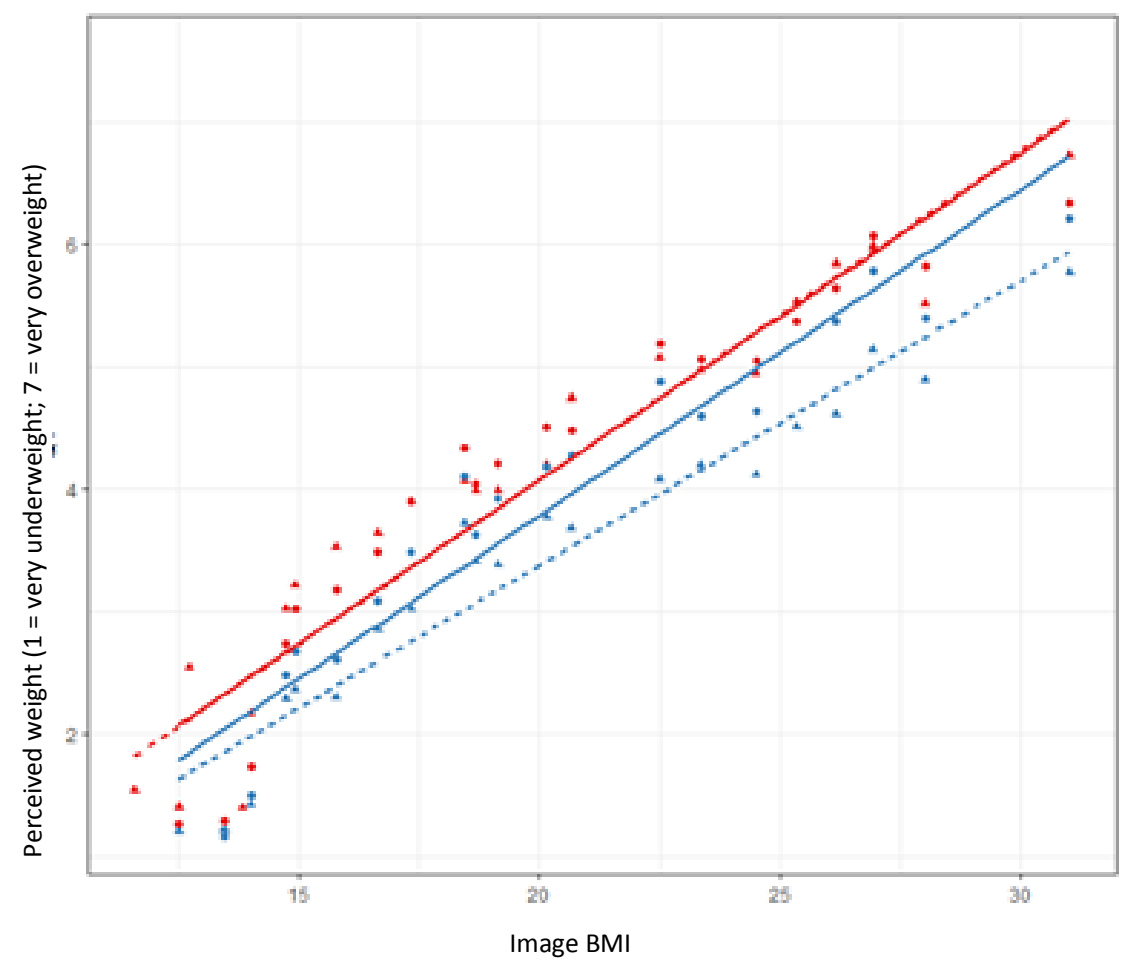

Figure 2. Mean weight rating given to each test image at pre- and post-test for each group. Those adapted to thin bodies are shown in red, those adapted to larger bodies are shown in blue. Pre-test data indicated by circles and solid regression line; post-test data indicated by triangles and dashed regression line.

Like Boothroyd et al (2018) the current study shows no evidence for an overall shift in the central point of reference for body perceptions, but rather a change in representations of bodies at one end of the weight spectrum versus the other. And like Boothroyd et al (2018) we find effects which are driven by exposure to larger bodies rather than low weight bodies. This may be explained by the over-representation of very slim bodies in British visual media, such that participants are already saturated with these stimuli. We note however, that other authors (e.g. Hummel et al., 2013; Winkler \& Rhodes, 2005) have sometimes found it easier to manipulate body perceptions with slim stimuli than with larger bodies. It is possible that differences in stimuli may contribute to this (it is unlikely that participants in Australia are fundamentally different to those in the UK or China in terms of general visual diet).

A key implication of our results is that those visual processes which maintain thin ideals in adult populations also likely apply to children from early puberty onwards and that any remedial changes in our visual environment (e.g. inclusion of a more representative sample of body weights in the media) would likely benefit these younger groups to the same degree as adults. This is also consistent with evidence showing that thin ideal internalisation (perception of and reliance on media images as ones standard for physical ideals) is evident from 6 or 7 years upwards (e.g. Evans et al., 2013; OTHERS), and the limited evidence that viewing images of slim bodies (e.g. Barbie dolls) may induce a desire for a slimmer body in young girls (Dittmar et al., 2006).

One caveat regarding our data is that by relying on the Psychology participant pool for our adult sample, we had significantly fewer male participants in this upper age bracket and were unable to use gender in interactions; the lack of a gender or age group difference in ratings, however, suggests that gender is unlikely to be a moderating factor in these results. We also note that our data were gathered within a small geographical area and strongly recommend replication with more representative international samples. Finally, it is essential that this approach is also extended into 
younger participants insofar as broader task demands allow in order to verify at what age these adaptation effects can first be seen to be adult-like.

In conclusion, we have demonstrated that perceptions of body weight are subject to adaptation after-effects which are adult-like from 11 years of age onwards, and that these effects are more pronounced for test images closer in weight to the adapted-extreme. These results thus have implications for our understanding of body size (mis)perception in health and wellbeing contexts as well as for our broader understanding of the development of body perception.

\section{References}

Anschutz, D. J., \& Engels, R. C. (2010). The effects of playing with thin dolls on body image and food intake in young girls. Sex roles, 63(9-10), 621-630.

Anzures, G., Mondloch, C. J., \& Lackner, C. (2009). Face Adaptation and Attractiveness Aftereffects in 8-Year-Olds and Adults. Child Development, 80(1), 178-191. doi:10.1111/j.1467-8624.2008.01253.x

Douglas Bates, Martin Maechler, Ben Bolker, Steve Walker (2015). Fitting Linear Mixed-Effects Models Using Ime4. Journal of Statistical Software, 67(1), 1-48. doi:10.18637/jss.v067.i01.

Boothroyd, L. G., Tovee, M. J., \& Pollet, T. V. (2012). Visual Diet versus Associative Learning as Mechanisms of Change in Body Size Preferences. Plos One, 7(11), e48691. doi:10.1371/journal.pone.0048691

Boothroyd, L.G., Pollet, T.V., Qu, Y., Evans, E.H. \& Tovee, M.J. (2018). Adaptation-like effects in body weight attractiveness are not simply norm based. Scientific Reports, submitted.

Brooks, K. R., Mond, J. M., Stevenson, R. J., \& Stephen, I. D. (2016). Body Image Distortion and Exposure to Extreme Body Types: Contingent Adaptation and Cross Adaptation for Self and Other. Frontiers in Neuroscience, 10, 334. doi:10.3389/fnins.2016.00334

Connolly, J. M., Slaughter, V., \& Mealey, L. (2004). The development of preferences for specific body shapes. Journal of Sex Research, 41(1), 5-15.

Dennett, H. W., McKone, E., Edwards, M., \& Susilo, T. (2012). Face aftereffects predict individual differences in face recognition ability. Psychological Science, 23(11), 1279-1287.

Dittmar, H., Halliwell, E., \& Ive, S. (2006). Does Barbie make girls want to be thin? The effect of experimental exposure to images of dolls on the body image of 5-to 8-year-old girls. Developmental psychology, 42(2), 283.

Evans, E. H., Tovée, M. J., Boothroyd, L. G., \& Drewett, R. F. (2013). Body dissatisfaction and disordered eating attitudes in 7-to 11-year-old girls: Testing a sociocultural model. Body Image, 10(1), 8-15.

Fontan, A., Cignetti, F., Nazarian, B., Anton, J. L., Vaugoyeau, M., \& Assaiante, C. (2017). How does the body representation system develop in the human brain?. Developmental cognitive neuroscience, 24, 118-128.

Fu, G. Y., Mondloch, C. J., Ding, X. P., Short, L. A., Sun, L. P., \& Lee, K. (2014). The neural correlates of the face attractiveness aftereffect: A functional near-infrared spectroscopy (fNIRS) study. Neuroimage, 85, 363-371. doi:10.1016/j.neuroimage.2013.04.092

Glauert, R., Rhodes, G., Byrne, S., Fink, B., \& Grammer, K. (2009). Body dissatisfaction and the effects of perceptual exposure on body norms and ideals. International Journal of Eating Disorders, 42(5), 443-452.

Hlavac, Marek (2018). stargazer: Well-Formatted Regression and Summary Statistics Tables. R package version 5.2.1. https://CRAN.R-project.org/package=stargazer

Hummel, D., Rudolf, A. K., Brandi, M. L., Untch, K. H., Grabhorn, R., Hampel, H., \& Mohr, H. M. (2013). Neural Adaptation to Thin and Fat Bodies in the Fusiform Body Area and Middle Occipital Gyrus: An fMRI Adaptation Study. Human Brain Mapping, 34(12), 3233-3246. doi:10.1002/hbm.22135

Jellinek, R. D., Myers, T. A., \& Keller, K. L. (2016). The impact of doll style of dress and familiarity on body dissatisfaction in 6-to 8-year-old girls. Body image, 18, 78-85.

Jiang, M. Y., \& Vartanian, L. R. (2016). The role of memory in the relationship between attention toward thin-ideal media and body dissatisfaction. Eating and Weight Disorders-Studies on Anorexia, Bulimia and Obesity, 21(1), 57-64.

Kadosh, K. C., Johnson, M. H., Henson, R. N., Dick, F., \& Blakemore, S. J. (2013). Differential face-network adaptation in children, adolescents and adults. Neuroimage, 69, 11-20.

McKone, E., Crookes, K., \& Kanwisher, N. (2009). The cognitive and neural development of face recognition in humans. The cognitive neurosciences, 4, 467-482.

Nishimura, M., Maurer, D., Jeffery, L., Pellicano, E., \& Rhodes, G. (2008). Fitting the child's mind to the world: adaptive norm-based coding of facial identity in 8-year-olds. Developmental Science, 11(4), 620-627. doi:10.1111/j.14677687.2008.00706.x

Peelen, M. V., B. Glaser, P. Vuilleumier and S. Eliez (2009). "Differential development of selectivity for faces and bodies in the fusiform gyrus." Developmental Science 12(6): F16-25.

Pelphrey, K. A., J. Lopez and J. P. Morris (2009). "Developmental continuity and change in responses to social and nonsocial categories in human extrastriate visual cortex." Frontiers in human neuroscience 3.

R Core Team (2018). R: A language and environment for statistical computing. R Foundation for Statistical Computing, Vienna, Austria. URL https://www.R-project.org/.

Rice, K., Prichard, I., Tiggemann, M., \& Slater, A. (2016). Exposure to Barbie: Effects on thin-ideal internalisation, body esteem, and body dissatisfaction among young girls. Body Image, 19, 142-149.

Robinson, E., \& Kirkham, T. C. (2014). Is he a healthy weight? Exposure to obesity changes perception of the weight status of others. International Journal of Obesity, 38(5), 663. 
Ross, P. D., B. de Gelder, F. Crabbe and M.-H. Grosbras (2014). "Body-Selective Areas in the Visual Cortex are less active in Children than in Adults." Frontiers in Human Neuroscience 8.

Ross, P., de Gelder, B., Crabbe, F. and Grosbras, M-H. (submitted) Emotion modulation of the body -selective areas in the developing brain.

Stephen, I. D., Bickersteth, C., Mond, J., Stevenson, R. J., \& Brooks, K. R. (2016). No effect of featural attention on body size aftereffects. Frontiers in psychology, 7, 1223.

Stephen, I. D., Sturman, D., Stevenson, R. J., Mond, J., \& Brooks, K. R. (2018). Visual attention mediates the relationship between body satisfaction and susceptibility to the body size adaptation effect. PloS one, $13(1)$, e0189855.

Stephen, I. D., \& Perera, A. T. M. (2014). Judging the differences between women's attractiveness and health: Is there really a difference between judgments made by men and women? Body Image, 11(2), 183-186. doi:10.1016/j.bodyim.2013.11.007

Sturman, D., Stephen, I. D., Mond, J., Stevenson, R. J., \& Brooks, K. R. (2017). Independent Aftereffects of Fat and Muscle: Implications for neural encoding, body space representation, and body image disturbance. Scientific Reports, 7, 40392. doi:10.1038/srep40392

Susilo, T., Germine, L., \& Duchaine, B. (2013). Face recognition ability matures late: Evidence from individual differences in young adults. Journal of experimental psychology: human perception and performance, 39(5), 1212

Tovee, M. J., \& Cornelissen, P. L. (2001). Female and male perceptions of female physical attractiveness in front-view and profile. British Journal of Psychology, 92, 391-402.

Tovee, M. J., Maisey, D. S., Emery, J. L., \& Cornelissen, P. L. (1999). Visual cues to female physical attractiveness. Proceedings of the Royal Society of London Series B-Biological Sciences, 266(1415), 211-218.

Winkler, C., \& Rhodes, G. (2005). Perceptual adaptation affects attractiveness of female bodies. British Journal of Psychology, 96, 141-154. doi:10.1348/000712605×36343

Supplementary Table 1. Mixed effect model with Time $x$ Group x Image BMI interaction specified separately for each age group.

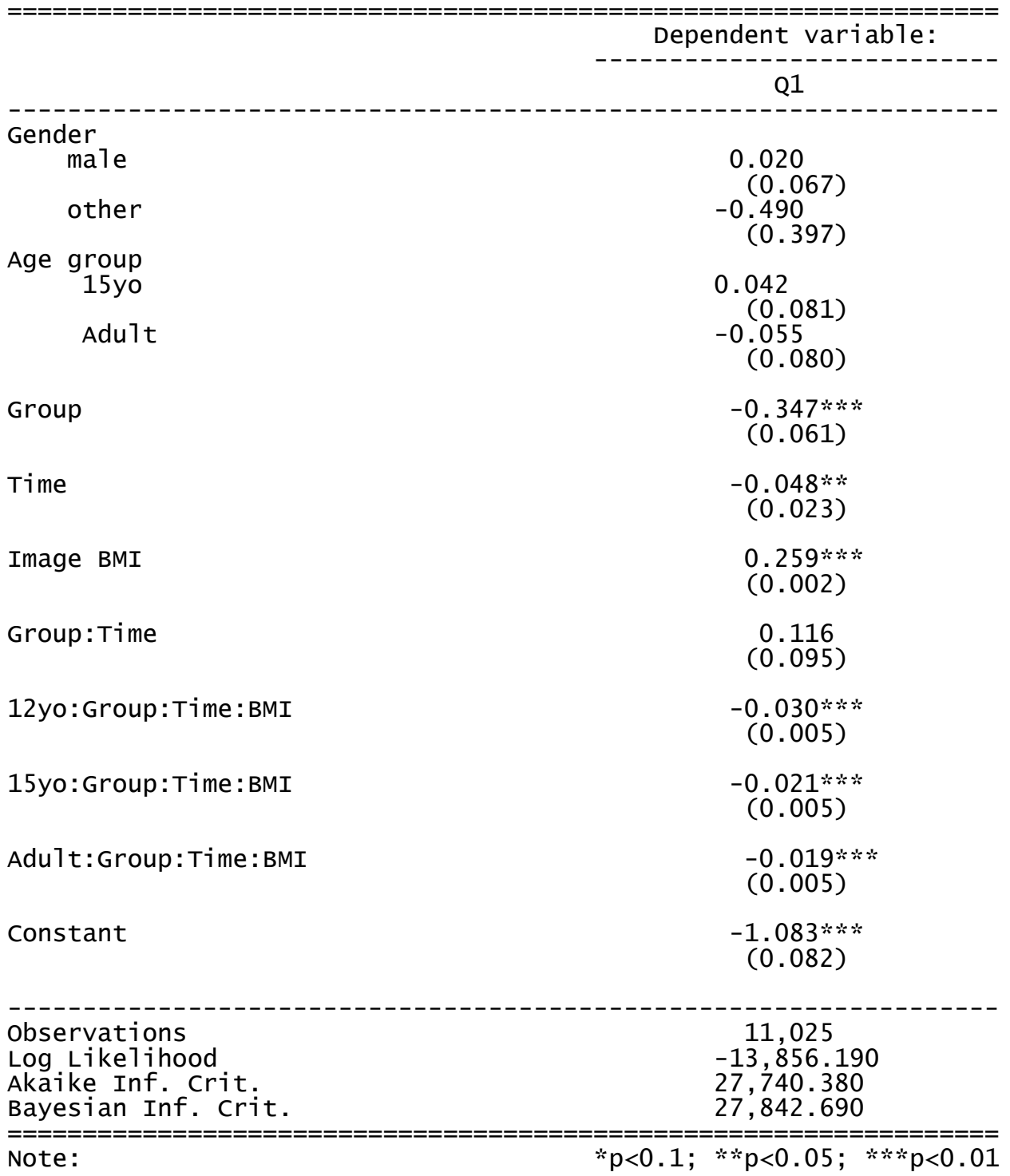

